\title{
Le tre rivoluzioni del management digitale
}

\author{
Stefano Micelli
}

\section{Le rivoluzioni del digitale}

Nel corso degli ultimi anni si è ampiamente diffusa l'abitudine di definire gli importanti cambiamenti economici in atto come una vera e propria "rivoluzione digitale". L'innovazione tecnologica innescata da informatica e telecomunicazioni ha contribuito a stravolgere radicalmente le forme di accesso all'informazione, i modelli di organizzazione della produzione e i modi del consumo. L'importanza di questi cambiamenti e la loro pervasività giustifica pienamente l'utilizzo della parola "rivoluzione" anche se l'utilizzo di questa espressione non aiuta di per sé uno sguardo analitico ai cambiamenti in atto, soprattutto per quanto riguarda le trasformazioni nella vita delle imprese. I cambiamenti che siamo chiamati a osservare sono molteplici e il richiamo ad una grande (unica) rivoluzione digitale non agevola chi vuole considerare in maniera più dettagliata l'impatto delle diverse famiglie di tecnologie nei processi economici.

Nell'ambito delle discipline manageriali, l'utilizzo dell'espressione "rivoluzione" ha segnato tre grandi momenti di trasformazione tecnologica, ciascuno dei quali caratterizzato dall'introduzione di strumenti e principi gestionali che hanno trasformato aree specifiche dell'azione di impresa. Queste tre diverse ondate tecnologiche hanno contribuito a ridefinire, in momenti diversi, le leve e le modalità operative con cui le imprese si confrontano con il mercato e definiscono i propri assetti organizzativi. Per riflettere sul cambiamento di paradigma cui è chiamato il management delle imprese è utile considerare ciascuna singola fase evolutiva tenendo conto che la sovrapposizione fra queste diverse spinte contribuisce ad accelerare le forme di governo delle imprese e, almeno in parte, i fondamenti della discipline manageriali.

Le tre rivoluzioni che hanno segnato l'evoluzione del management dai primi anni ' 90 ad oggi possono essere riassunte mettendo in evidenza le diverse tecnologie che hanno messo in moto importanti altrettanti processi di cambiamento e le aree di impresa che sono state oggetto di trasformazione. Nei primi anni '90 una prima rivoluzione manageriale prende forma con l'introduzione massiccia di nuove tecnologie per la gestione integrata di impresa (i sistemi gestionali di cui SAP è ancora il leader a livello mondiale). Queste tecnologie, spesso sottostimate per importanza nel dibattito italiano, hanno determinato una riorganizzazione sostanziale delle imprese di grande dimensione e hanno rappresentato l'infrastruttura gestionale per i processi di delocalizzazione produttiva e di internazionalizzazione dei mercati che hanno segnato un aspetto essenziale dei processi di globalizzazione economica negli ultimi vent’anni.

Una seconda rivoluzione tecnologica prende forma alla fine degli anni '90 e all'inizio del decennio successivo grazie alla diffusione del Web come 
sinergie Vol. 35, N. 103, 2017

strumento destinato a fini di comunicazione e commerciali. L'impatto di questi nuovi strumenti di comunicazione è legato principalmente al rapporto fra impresa e consumatori: in pochi anni il commercio elettronico assume una rilevanza crescente grazie a piattaforme che assumono rilevanza globale. Si modifica, inoltre, il modo di gestire i processi di comunicazione fra impresa e consumatore finale grazie a nuovi strumenti di comunicazione che modificano in chiave bidirezionale processi comunicativi a lungo gestiti in forma unidirezionale. Anche in questo caso l'espressione "rivoluzione" è legittima perché le nuove modalità di organizzazione dei rapporti fra impresa e mercato innescano una ridefinizione complessiva dei principi e delle pratiche operative che la funzione del marketing aveva consolidato nel tempo.

Una terza rivoluzione tecnologica prende forma in questi anni grazie all'introduzione del digitale nell'ambito dei processi produttivi in senso stretto. La diffusione di tecnologie per la manifattura additiva (stampanti $3 \mathrm{D})$ e di robot a costi sempre più contenuti segna un nuovo passo in avanti nell'introduzione del digitale nelle attività di impresa. Queste nuove tecnologie hanno un impatto rivoluzionario non solo perché contribuiscono a ridefinire in modo sostanziale la geografia della produzione a livello mondiale ma anche perché introducono elementi di novità a livello di strategie di impresa mettendo a disposizione delle imprese la possibilità di personalizzare il prodotto a livelli mai sperimentati in precedenza.

In che modo la combinazione di queste tre spinte convergenti contribuiscono a ridefinire in modo complessivo il funzionamento delle imprese, la definizione delle loro strategie competitive, le forme di organizzazione del lavoro? È presto per formulare affermazioni definitive. Ciò che è alla nostra portata è prendere in esame le principali traiettorie evolutive innescate da queste "rivoluzioni parallele" valutando il loro impatto in termini manageriali.

Questo esercizio, è importante sottolinearlo, non può essere svolto in astratto ma va riportato alle specificità dei contesti nazionali, in particolare per quelle realtà come l'Italia, dove i percorsi di sviluppo delle imprese hanno assunto caratteristiche specifiche che ora dovranno misurarsi con i grandi cambiamenti imposti dal digitale.

\section{La riorganizzazione dei processi di impresa (BPR)}

La prima vera e propria rivoluzione manageriale legata all'introduzione del digitale nelle imprese coincide con l'introduzione dei cosiddetti sistemi di gestione integrata come infrastruttura digitale per il management delle imprese. Queste tecnologie hanno rappresentato un importante salto tecnologico rispetto alle tradizionali strumentazioni informatiche disponibili presso le imprese fino alla fine degli anni ' 80 .

L'informatica aziendale aveva sviluppato fino a quella data soluzioni software capaci di dare risposte a specifiche funzioni aziendali (l'amministrazione, la finanza, la produzione, il marketing, etc.) senza che fosse possibile contare su basi di dati condivise e senza poter contare su soluzioni semplici per la gestione di processi interfunzionali. Inoltre, i 
software disponibili costituivano una base di partenza per la creazione di Stefano Micelli soluzioni "taylor made" che rendevano le diverse declinazioni di un singolo pacchetto applicativo molto diverse fra loro. I software per la gestione integrata di impresa, in particolare il leader mondiale del settore SAP, ribaltano questa logica di sviluppo proponendo una unica piattaforma con diverse applicazioni per le diverse funzioni aziendali e un'unica base di dati di riferimento (Camussone, 1998). I gestionali integrati, inoltre, lasciano all'utilizzatore finale una discrezionalità di utilizzo contenuta: le funzioni aziendali possono ancora modificare il codice del sistema, ma gran parte dell'effettivo utilizzo del sistema passa per semplici procedure di parametrizzazione dell'applicativo. Non si compra solo software, si compra intelligenza gestionale (Micelli, 2000).

L'introduzione di queste soluzioni di gestione integrata innesca un profondo cambiamento nel funzionamento delle dinamiche organizzative delle grandi imprese multinazionali che le riviste scientifiche e le società di consulenza battezzano come Business Process Reengineering (BPR). Questa trasformazione coincide con un cambiamento sostanziale nelle modalità di gestione delle organizzazioni che, grazie alle tecnologie, superano il modello ordinato per funzioni e adottano un modello strutturato per processi. Le piattaforme di gestione integrata diventano in pochi anni il grimaldello per sbloccare le burocrazie interne alle grandi corporation americane e europee che avevano limitato in modo considerevole la competitività delle grandi imprese.

Un breve cenno storico contribuisce a dare rilevanza alla dimensione "rivoluzionaria" dell'introduzione di queste tecnologie. All'inizio degli anni '90 il dibattito manageriale e l'attività delle maggiori società di consulenza a livello internazionale sono concentrati sulla diffusione del Total Quality Management al di fuori del perimetro delle principali aziende giapponesi che lo avevano sviluppato e che lo hanno reso famoso (Toyota in primis). A cavallo fra anni ' 80 e anni '90 si discute della necessità di come rimettere il lavoratore al centro dei processi di miglioramento continuo, di come evitare gli sprechi attivando processi decisionali distribuiti (lean management), di come passare da modalità gestionali in logica push a criteri incardinati sull'effettivo comportamento della domanda.

Dopo decenni di management di matrice tecnocratica ereditato dall'esperienza della grande corporation americana, il modello giapponese del Total Quality Management aveva il merito di assegnare valore a forme di cambiamento partecipate e graduali, a processi di miglioramento che facevano diventare lo spazio della produzione un campo di innovazione centrato sull'attenzione e la motivazione dei dipendenti. La declinazione del modello giapponese non è mai passata attraverso l'implementazione forzosa di tecnologie sofisticate, puntando piuttosto alla semplificazione e al confronto diretto fra i singoli lavoratori (basti pensare all'uso del kanban, il cartellino posto sui moduli di trasporto del magazzino per gestire la linea di produzione secondo modalità pull).

La pubblicazione del volume di Hammer e Champy (1994), che nel titolo esplicitamente parla di una "rivoluzione" nelle logiche di organizzazione aziendale, e di molti altri articoli che segnano il dibattito di quegli anni, propone un cambiamento di prospettiva sostanziale. Questa letteratura 
sinergie Vol. 35, N. 103, 2017

sottolinea in modo esplicito la dimensione radicale del cambiamento imposto dalle nuove tecnologie e propone metodologie di intervento che puntano a implementare forme radicalmente nuove di organizzazione per processi. Le strutture aziendali delle grandi imprese americane, segnate da grandi burocrazie interne costruite attorno a funzioni silos incapaci di dialogare in modo funzionale, scoprono il potenziale di strumenti che aumentano radicalmente la produttività e la trasparenza dei loro processi interni ed esterni. Lorizzonte di un miglioramento continuo a partire dallesistente fa spazio a un'idea di discontinuità tecnologica che propone il sovvertimento dei tradizionali criteri di organizzazione manageriale.

A livello internazionale, la diffusione di queste tecnologie sostiene e acceleraiprocessi di riorganizzazione ascalaglobale digrandiorganizzazioni che, proprio grazie alle nuove tecnologie, possono organizzare catene del valore a scala globale. I nuovi sistemi gestionali consentono, soprattutto, di gestire processi di fornitura a scala internazionale fornendo il supporto operativo per quelle dinamiche di offshoring che hanno segnato l'ultimo decennio del secolo scorso e quello successivo. La velocità e la qualità nello scambio delle informazioni ha permesso alle imprese di maggiori dimensioni di modulare le forme di governance della fornitura internazionale consentendo importanti miglioramenti di efficacia e di efficienza a quelle imprese che hanno saputo sviluppare forme evolute di global value chains (Gereffi et al., 2005; Chiarvesio et al., 2010).

In Italia, l'introduzione delle tecnologie per la gestione integrata di impresa non hanno avuto lo stesso impatto ottenuto negli Stati Uniti o in altri paesi europei perché, rispetto al messaggio di Hammer e Champy, non cera una corporation da ridisegnare. La fine degli anni '80 e l'inizio degli anni '90 rappresentano per il nostro paese il momento del massimo successo della formula dei distretti industriali e della competitività della piccola impresa. Pochi anni prima autori come Sabel e Piore avevano celebrato il modello italiano come alternativa al fordismo tradizionale, come l'esempio di una dinamica di sviluppo capace di saldare società e crescita grazie a al modello della "specializzazione flessibile" (Piore e Sabel, 1985). Le piccole imprese italiane avevano trovato la soluzione a un mercato più dinamico e differenziato grazie a unorganizzazione di tipo territoriale che non aveva particolare bisogno di nuove tecnologie per esprimere la sua competitività. Per questo, l'introduzione di queste tecnologie, arriverà solo con diversi anni di ritardo e riguarderà principalmente quelle medie imprese internazionalizzate che Unioncamere e Mediobanca hanno ribattezzato il "quarto capitalismo" italiano (a proposito si veda Serio, 2017).

\section{La rivoluzione del Web}

Vi sono pochi dubbi sul fatto che la rapida diffusione del Web abbia costituito una rivoluzione sostanziale nel modo di fare impresa, in particolare per quanto riguarda i rapporti fra impresa e consumatore. L'importanza di questi cambiamenti, in verità, non è mai stata sottostimata. Fin dalla fine degli anni '90 la diffusione della rete e i cambiamenti che 
Internet avrebbe innescato sono stati pensati come l'inizio di una vera e propria "New economy", con regole proprie, specifiche e diverse rispetto del management digitale a quelle che hanno regolato l'economia del passato. Non sono mancate, ovviamente, voci critiche a questa lettura radicale del cambiamento posto in essere dalla rete e dal Web; ciò detto, i cambiamenti sperimentati negli ultimi vent'anni confermano retrospettivamente molte delle tesi avanzate da chi immaginava uno stravolgimento delle logiche del commercio e della comunicazione.

Va segnalato che molte delle riflessioni sui principali cambiamenti in atto non sono passate per i canali tradizionali del dibattito scientifico ma hanno trovato spazio soprattutto in una nuova generazione di riviste di taglio internazionale che hanno avuto il merito di imporre temi di grande attualità all'attenzione dell'opinione pubblica. Autori come Kevin Kelly, saggista e editorialista di Wired, come John Seely Brown, a lungo direttore del prestigioso Xerox Parc, o John Hagel, importante consulente di McKinsey, hanno saputo impostare un dibattito importante su alcune questioni essenziali riguardo alla discontinuità con il paradigma manageriale che abbiamo lasciato alle nostre spalle. Molte di queste idee per gestire "un mondo turbolento" hanno segnato una rottura netta con assunti consolidati a livello di marketing e di gestione dell'innovazione. Questi e molti altri autori hanno contribuito allo spostamento di attenzione dal mondo delle tecnologie (al tempo etichettato information and communication technologies) alla dimensione del digitale, espressione con la quale si definisce non solo lo strumento tecnologico ma anche e soprattutto la nuova cultura che lo strumento è in grado di abilitare.

Alcune delle leggi promosse da Kelly (1998), in particolare, sono ancora oggi di grande attualità. Le sue suggestioni su un mondo completamente interconnesso, capace di produrre intelligenza in forme nuove e non necessariamente centralizzate, sono ancora particolarmente attuali anche se oggi il dibattito mediatico etichetta il fenomeno come "Internet delle cose". Le possibilità di un mondo totalmente interconnesso aprono nuove opportunità non solo nei settori ad alta intensità tecnologica ma anche nei settori tradizionali (basti pensare agli attuali avanzamenti della cosiddetta agricoltura di precisione). Le sue intuizioni sui ritorni crescenti dei network hanno rappresentato un punto di svolta nel cogliere il valore di tante start up promettenti e nel definire le strategie di penetrazione di tante imprese attive sul Web. Oggi siamo tutti consapevoli della necessità di costruire network e ecosistemi in cui l'impresa può crescere e prosperare e siamo consapevoli che l'avvio di questi ecosistemi richiede una "generosità" iniziale cui le imprese tradizionali non sono state abituate.

La crescita del Web ha innescato lo sviluppo di una nuova generazione di imprese piattaforma che hanno saputo costruire percorsi di crescita originali rispetto alle grandi imprese che le hanno precedute. La crescita straordinaria di questi nuovi protagonisti del settore tecnologico, da Amazon a Facebook, da Google a AirB\&B, ha profondamente ridefinito il settore di riferimento e ha costretto ad una rapida riorganizzazione anche settori apparentemente lontani dal digitale in senso stretto, dalla musica alleditoria, dal turismo al commercio al dettaglio. 
sinergie Vol. 35, N. 103, 2017

Oggi il successo di questi campioni del Web, ha superato di molto le aspettative iniziali e ha contribuito a modificare la percezione del ruolo di questi campioni nello scenario internazionale. Se fino a pochi anni fa queste imprese godevano di un pregiudizio positivo nel mondo della ricerca così come presso l'opinione pubblica, oggi questo percepito è rapidamente mutato lasciando spazio a timori - fondati - che la posizione di monopolio di fatto esercitato da queste imprese possa trasformarsi in un vincolo alla concorrenza e a una sperequazione della distribuzione del valore.

Limpatto generato dalla rete sulle imprese tradizionali ha riguardato principalmente il loro modo di confrontarsi con la domanda finale. La facilità di accesso alle informazioni e la possibilità di condividere in modo informale giudizi e valutazioni su prodotti e servizi ha contribuito a spostare in modo irreversibile il baricentro del potere negoziale fra produttore e acquirente.

Quest'ultimo, lungi dall'essere solo e isolato nei suoi processi decisionali, può far leva su connessioni sociali digitali che gli consentono una capacità di valutazione e di proposta prima sconosciute. Questo stesso patrimonio di conoscenze e relazioni rappresenta per le imprese unopportunità di crescita e di innovazione (Hagel e Armstrong, 1997).

Le comunità digitali con cui l'impresa è chiamata a confrontarsi sono spesso capaci di formulare proposte, contribuire al miglioramento dei prodotti, suggerire spazi di crescita e di sperimentazione. Se il consumatore tradizionale delegava all'impresa il compito di innovare e di definire standard plausibili per l'evoluzione del prodotto reclamando per sé la possibilità di scegliere fra opzioni diverse, il nuovo consumatore - non più isolato - si immagina come parte attiva del processo di creazione del valore attraverso la partecipazione a comunità attive, spesso relativamente autonome dai brand di riferimento. Il nuovo consumatore vuole conoscere, commenta sulle piattaforme dei social network, prende posizione, rilancia commenti positivi e negativi. Chiede, in altre parole, un dialogo e un'interazione che solo le nuove tecnologie rendono economicamente sostenibile.

Le nuove regole di interazione con il mercato hanno profondamente influenzato il modo di comunicare il Made in Italy. Dopo alcuni anni di incertezza, le imprese italiane hanno avviato un percorso evolutivo che ha consentito di mettere a punto soluzioni di comunicazione originale verso la domanda interna e, soprattutto verso la domanda internazionale.

La natura stessa di molti beni prodotti in Italia, beni di nicchia dal forte potere simbolico, ha innescato forme di autoorganizzazione della domanda a prescindere dalle azioni intraprese dal management. Se è vero che molta strada rimane ancora da fare, è altrettanto vero che molte imprese italiane, anche di piccole dimensioni hanno dimostrato di saper cogliere le opportunità offerte da nuove logiche di comunicazione bidirezionale (Bettiol, 2015).

\section{La rivoluzione della manifattura digitale}

Nel corso degli ultimi cinque anni, il tema del digitale ha assunto una nuova valenza. Dopo aver investito e ridefinito la dimensione gestionale e amministrativa delle imprese, grazie all'introduzione dei sistemi di 
gestione integrata, e dopo aver trasformato radicalmente le funzioni di interfaccia fra organizzazione e mercato, grazie al Web e ai nuovi canali di comunicazione messi a disposizione da Internet, le nuove tecnologie contaminano oggi i processi manifatturieri in senso stretto.

La manifattura digitale non è un fatto radicalmente nuovo. Molte delle tecnologie che oggi consideriamo abilitanti di un nuovo paradigma tecnologico sono in realtà presenti sul mercato da diversi anni. Le macchine a controllo numerico sono state sviluppate negli anni ' 50 e sono diventate parte della dotazione tecnologica delle imprese di produzione. I dispositivi per il taglio laser sono stati inventati negli anni '70 e hanno conosciuto una diffusione nelle imprese già dagli anni '80. Anche le stampanti 3D, nelle loro diverse versioni, sono presenti sul mercato dalla fine degli anni '80 e sono entrate a far parte della dotazione dei laboratori di prototipazione già durante gli anni '90. Non è la novità in senso stretto di queste tecnologie a rendere queste tecnologie "rivoluzionarie" quanto piuttosto la loro diffusione a basso costo, fenomeno piuttosto recente, e la possibilità di sfruttare il loro potenziale tecnologico attraverso strumenti di programmazione relativamente accessibili.

Economicità e accessibilità delle tecnologie rendono più facile e "democratico" (Von Hippel, 2005) l'accesso a strumenti sofisticati in grado di collegare il mondo della progettazione informatica (il CAD) e il mondo della produzione in senso stretto (il CAM). Proprio questa democraticità di utilizzo ha messo in moto dinamiche nuove di organizzazione della produzione: se è vero che hanno consentito alle imprese di maggiori dimensioni di investire se questi strumenti a una scala estensiva rispetto al passato (rilanciando il progetto di "fabbrica automatica" che aveva acquisito visibilità alla fine degli anni '80) è altrettanto vero che hanno permesso a nuovi soggetti e a imprese di dimensione limitata di acquisire strumenti un tempo inaccessibili (Anderson, 2012).

Come tradurre queste nuove opportunità tecnologiche in valore economico? Neil Gershenfeld, fondatore del primo Fab Lab presso il MIT di Boston, è stato fra i primi a fornire risposte a questa domanda elaborando una serie di indicazioni a cavallo fra tecnologia e economia. Gershenfeld sottolinea che il principale beneficio generato dalla diffusione delle tecnologie è quello di aumentare radicalmente il livello di personalizzazione degli oggetti prodotti grazie ai nuovi strumenti della manifattura digitale. La combinazione di questi strumenti consente di produrre praticamente qualsiasi cosa, parafrasando il titolo del celebre articolo "How to Make Almost Anything", superando i limiti tradizionali imposti dalle logiche tipiche della produzione di massa (Gershenfeld, 2012). Anche in passato, in verità, era possibile personalizzare oggetti sofisticati facendo riferimento ad artigiani che svolgevano la loro attività nell'ambito di nicchie di attività specifiche, lontane dalle logiche di una domanda standardizzata. Questa attività di personalizzazione, tuttavia, era destinata solo a un consumatore con ampie disponibilità di spesa e in grado di aspettare il tempo necessario per la realizzazione di un determinato prodotto.

La tesi di Gershenfeld è che il trade off tradizionale fra l'accessibilità economica di un bene e la sua personalizzazione (più un bene è standardizzato più è economico, più un bene e personalizzato più è 
sinergie Vol. 35, N. 103, 2017

costoso) venga meno perché le nuove tecnologie rendono possibile la sua produzione personalizzata a costi tipici del modello di produzione in scala. La diffusione di queste tecnologie, se combinata a una rapida socializzazione delle conoscenze necessarie al loro effettivo utilizzo, può rappresentare la chiave di volta di un nuovo modo di pensare i processi produttivi. Non sono necessariamente le imprese di tipo tradizionale a diventare il motore di questo processo di diffusione delle conoscenze e di questa manifattura digitale: anche infrastrutture come i Fab Lab, i Makerspace, o altri spazi per la socializzazione di soluzioni tecnologiche avanzate (incubatori, acceleratori, altro) possono giocare un ruolo fondamentale nella diffusione di una nuova cultura tecnologica e, in seconda battuta, nella messa a punto di infrastrutture comuni per la produzione all'interno di una specifica comunità (Hirshberg et al., 2016).

Mentre negli Stati Uniti le ipotesi di una tecnologia in grado di svilupparsi rapidamente grazie a dinamiche di partecipazione bottom up hanno ricevuto un solido consenso istituzionale, in Europa la diffusione delle nuove tecnologie per la manifattura digitale ha beneficiato di iniziative di maggiore consistenza istituzionale. I progetti a sostegno di un percorso di innovazione rivolto all'"Industria 4.0" hanno caratterizzato prima la Germania, particolarmente attenta al fenomeno fin dal 2011, e successivamente diversi paesi europei fra cui anche l'Italia. Le iniziative di politica industriale promosse in Europa e in particolare nel nostro paese si sono caratterizzate per incentivi fiscali che hanno, da un lato, agevolato lacquisto di nuovi macchinari e di nuove tecnologie da parte di imprese già presenti sul mercato e che, dall'altro, hanno sostenuto la creazione di centri eccellenza e di percorsi formativi innovativi. Se negli Stati Uniti la rivoluzione tecnologica è pensata nei termini di nuove soggettività che si affacciano (spesso di prepotenza) sul mercato, nel contesto europeo queste trasformazioni vengono affidate a chi già oggi svolge un ruolo economico consolidato.

\section{Il risultato di trasformazioni convergenti}

Le tre grandi trasformazioni che il digitale ha impresso sulla gestione delle imprese hanno contribuito a mettere in discussione i tradizionali paradigmi dellazione manageriale. Come evidenziato in precedenza, il passaggio da modelli organizzativi basati su funzioni a modelli organizzativi centrati su processi organizzativi (e inter-organizzativi) grazie all'utilizzo di tecnologie per la gestione integrata di impresa ha rappresentato un primo importante cambiamento di paradigma. Grazie alla tecnologia, la grande corporation americana ha scoperto la possibilità di aumentare radicalmente la propria flessibilità strategica e operativa coinvolgendo nel proprio network soggetti terzi attivi in ogni parte del mondo grazie al contenimento strutturale dei costi di transazione. In questo nuovo scenario, la competitività delle imprese non dipende più semplicemente dalla strategia dell'impresa in senso stretto, quanto piuttosto dalla competitività della filiera all'interno della quale l'impresa opera e dal business model che l'impresa è in grado di mettere a punto. 
L'importanza del Web e di Internet nella riorganizzazione dei processi Stefano Micelli
Le tre rivoluzioni di relazione con il mercato ha reso questa dinamica ancora più stringente. del management digitale Secondo parte rilevante del pensiero manageriale legato alla new economy il compito dell'imprenditore è legato principalmente alla creazione e all'alimentazione di complessi ecosistemi da cui dipende il successo dell'impresa. Lazione manageriale è costretta a confrontarsi con dinamiche sistemiche che sono in buona sostanza estranee o solo in parte dipendenti dal controllo dell'imprenditore e della direzione di impresa. La possibilità di prosperare in nuovi ecosistemi complessi dipende dallabilità del management di governare processi interni e dinamiche di sistema esterne al perimetro proprietario dell'organizzazione.

La terza ondata tecnologica, riconducibile all'introduzione delle tecnologie per la manifattura digitale, mette a sua volta in discussione uno dei perni essenziali della teoria organizzativa ovvero l'assunto à la Thompson dell'esistenza di un nucleo tecnico (il processo produttivo con le sue economie di scala) da proteggere e stabilizzare grazie a funzioni manageriali in grado di assorbire le fonti di incertezza presenti nell'ambiente in cui l'impresa opera. I modelli organizzativi hanno a lungo immaginato l'impresa come un sistema a più strati: a fronte di processi produttivi da ottimizzare e da rendere stabili nel tempo, l'impresa mette in campo strutture di interfaccia con l'ambiente circostante finalizzate a limitare i rischi economici e cogliere le opportunità di mercato. In questa prospettiva, il management è la funzione in grado di arbitrare fra la complessità dell'ambiente e le stabilità di un nucleo tecnico da cui dipende l'economicità dell'impresa (Thompson, 1991).

Ciò che la letteratura suggerisce è proprio il venir meno di un nucleo tecnico di tipo tradizionale così come lo abbiamo ereditato dalla seconda rivoluzione industriale. I processi produttivi tradizionali hanno necessitato importanti investimenti in macchinari e procedure che hanno garantito una produzione standardizzata su larga scala. Le funzioni di management, dalla logistica al marketing si sono adeguate a questo sistema di vincoli creando strumenti per la gestione dei magazzini, tecniche di ottimizzazione degli impianti, campagne di comunicazione e vendita coerenti. I fautori della manifattura digitale promettono una flessibilità inaudita a livello di processi produttivi, grazie all'utilizzo di tecnologie universali che non richiedono particolari tempi e costi di attrezzaggio. Il management, che per un secolo ha "costretto" e limitato le aspettative della domanda entro vincoli determinati dalle rigidità dei processi produttivi, si trova ora a giocare un ruolo completamente nuovo essendo chiamato a valorizzare il potenziale di varietà e personalizzazione legato alla tecnologia.

La velocità e l'intensità di queste diverse ondate hanno messo in discussione diversi presupposti della teoria economia e del management. Difficile immaginare che un'unica riflessione possa includere fenomeni così eterogenei e in divenire (i cambiamenti nei processi decisionali, le nuove forme del lavoro, le trasformazioni dei processi produttivi, l'evoluzione della domanda) all'interno di un quadro teorico. Appare utile, tuttavia, sottolineare l'importanza e la relativa autonomia delle tre ondate tecnologiche per capire in che modo esse hanno concorso alla trasformazione dei modelli di management. Fare riferimento a una generica rivoluzione digitale non ha 
sinergie italian journal of management

particolare valore analitico. Rischia di rendere poco leggibili le relazioni di causa e effetto che legano fra loro trasformazioni tecnologiche e dinamiche gestionali. Più interessante identificare i driver che hanno determinato la rapida evoluzione sperimentata in questi anni e provare a mettere a fuoco la risultante delle forze in campo sull'azione di impresa.

\section{Bibliografia}

ANDERSON C. (2012), Makers: The New Industrial Revolution, Random House, New York.

BETTIOL M. (2015), Raccontare il Made in Italy, Marsilio, Venezia.

CAMUSSONE P.F. (1998), Il sistema informativo aziendale, Etas, Milano.

CHIARVESIO M., DI MARIA E., MICELLI S. (2010), "Global value chains and open networks: the case of Italian industrial districts", European Planning Studies, vol. 18, n. 3.

HIRSHBERG P., DOUGHERTY D., KADANOFF M. (2016), Maker City, Maker Media, San Francisco.

GEREFFI G., HUMPHREY J., STURGEON T. (2005), “The governance of global value chains", The Review of International Political Economy, vol. 12, n. 1.

GENSHERFELD N. (2012), "How to Make Almost Anything. The Digital Fabrication Revolution", Foreign Affairs, Nov.-Dec.

HAMMER M., CHAMPY J. (1994), Reengineering the Corporation. A Manifesto for Business Revolution, Harper Business, New York.

HAGEL J., ARMSTRONG A. (1997), Net Gain, Harvard University Press, Boston.

KELLY K. (1998), New Rules for the New Economy, Viking, New York.

MICELLI S. (2000), Imprese, reti, comunità virtuali, Etas, Milano.

PIORE M., SABEL C. (1986), The Second Industrial Divide: Possibilities For Prosperity, Basic Books, New York.

SERIO L. (2017), Medie eccellenti. Le imprese italiane nella competizione internazionale, Guerini e Associati, Milano.

THOMPSON J. (1991), L'azione organizzativa, ISEDI, Torino.

VON HIPPEL E. (2005), Democratizing Innovation, MIT Press, Cambridge Mass.

\section{Academic or professional position and contacts}

\section{Stefano Micelli}

Associate Professor of Management

University of Ca’ Foscari Venezia - Italy

e-mail: micelli@unive.it

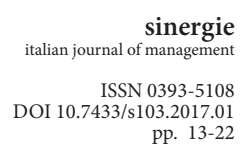

